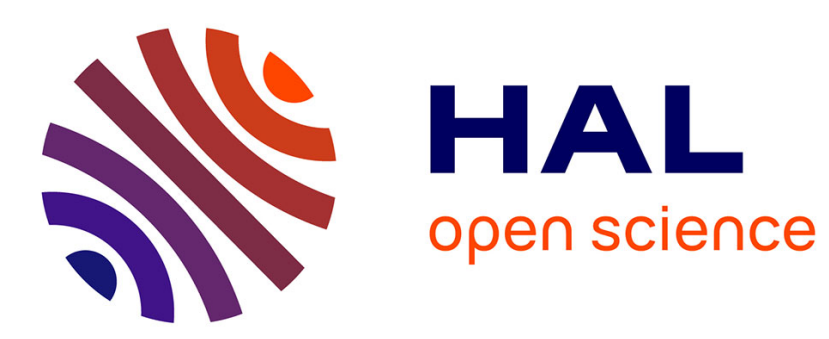

\title{
Resistivity of the Kondo lattice
}

\author{
M. Lavagna, Claudine Lacroix, Michel Cyrot
}

\section{To cite this version:}

M. Lavagna, Claudine Lacroix, Michel Cyrot. Resistivity of the Kondo lattice. Journal of Physics F: Metal Physics, 1982, 12 (4), pp.745-757. 10.1088/0305-4608/12/4/015 . hal-01896451

\section{HAL Id: hal-01896451 https://hal.science/hal-01896451}

Submitted on 16 Oct 2018

HAL is a multi-disciplinary open access archive for the deposit and dissemination of scientific research documents, whether they are published or not. The documents may come from teaching and research institutions in France or abroad, or from public or private research centers.
L'archive ouverte pluridisciplinaire HAL, est destinée au dépôt et à la diffusion de documents scientifiques de niveau recherche, publiés ou non, émanant des établissements d'enseignement et de recherche français ou étrangers, des laboratoires publics ou privés. 


\section{Resistivity of the Kondo lattice}

To cite this article: M Lavagna et al 1982 J. Phys. F: Met. Phys. 12745

View the article online for updates and enhancements.

\section{Recent citations}

\begin{tabular}{l} 
- Evidence for strong enhancement of the \\
$\frac{\text { magnetic ordering temperature of trivalent }}{\mathrm{Nd} \text { metal under extreme pressure }}$ \\
\hline $\mathrm{J}$ Song et al \\
- Impact of microstructure on the \\
$\frac{\text { thermoelectric properties of the ternary }}{\text { compound Ce3Cu3Sb4 }}$ \\
Piotr Witas et al \\
- Itinerant to local transformation and critical \\
point in Ni-rich $\mathrm{Ce}_{2}\left(\mathrm{Ni}_{4} \mathrm{Pd}_{4}\right)_{2} \mathrm{Sn}$ alloys \\
$\mathrm{J} \mathrm{G} \mathrm{Sereni}$ et al
\end{tabular}




\title{
Resistivity of the Kondo lattice
}

\author{
M Lavagna, C Lacroix and M Cyrot \\ Laboratoire Louis Néel (associé à l'Université Scientifique et Médicale de Grenoble), \\ CNRS, 166X, 38042 Grenoble Cedex, France
}

Received 5 August 1981

\begin{abstract}
The electrical resistivity of the Kondo lattice is studied within the model of Lacroix and Cyrot: in this model, the Kondo interaction is transformed into a fictitious $s-f$ hybridisation $J x / 2$. In order to describe the transport properties, we must go beyond a mean field theory and introduce the thermal fluctuations of the hybridisation $x$. At high temperatures (above $T_{K}$ ), the system may be treated as a collection of incoherent Kondo impurities. The resistivity, evaluated within the phase shift method, exhibits a logarithmic decrease as expected. At low temperature, coherence develops between impurities and the system is really a Kondo lattice. Spatial fuctuations (from site to site) of the $s-f$ hybridisation are considered as being responsible for the electron scattering. The resistivity depends strongly on the conduction electron number $n$ : we find for $n<1$ a metallic behaviour with a linear increase and a maximum at a temperature $T_{1} \sim(1-n)^{1 / 3} T_{\mathrm{K}}$ : for $n=1$ the resistivity is that of a semiconductor with $\rho \sim \exp \left(T_{\mathrm{K}} / T\right)$.
\end{abstract}

\section{Introduction}

The transport properties (resistivity, thermopower, Hall coefficient and magnetoresistance) of concentrated compounds with anomalous rare-earth fall into two categories (Lawrence 1981). Most ambivalent cerium and ytterbium based compounds exhibit metallic behaviour at low temperature, while samarium and thulium compounds exhibit activated conductivities in the ground state characteristic of a small gap semiconductor. In both cases, the high-temperature limit is that of Kondo impurities with a logarithmic decrease of the resistivity.

$\mathrm{CeAl}_{2}, \mathrm{CeIn}_{3}$ and $\mathrm{CePd}_{3}$ are typical examples of the first category with increasing resistivities when $T$ increases from $0 \mathrm{~K}$. The resistivity passes through a maximum at $T_{\max }$ before exhibiting the Kondo decrease in in $T$ at high temperature. The magnitude of the resistivity at high temperature or at $T_{\max }$ is often large due to spin-disorder scattering associated with a large Kondo exchange constant $J_{\text {sf }}$. These compounds also exhibit a positive thermopower maximum centred at a temperature comparable to the spin fluctuation temperature and of large magnitude.

$\mathrm{SmB}_{6}$, TmSe and probably M-SmS belong to the second category with an infinite resistivity at $T=0 \mathrm{~K}$ followed by a large decrease with temperature. This behaviour is characteristic of a small gap semiconductor. A particularly interesting 
case is provided by $\operatorname{Tm}_{x} \mathrm{Se}$ : out of stoichiometry, the compound exhibits metallic behaviour at low temperature while the residual $T=0$ resistivity diverges as stoichiometry is approached.

Many authors agree that these transport properties must find an explanation in the Kondo lattice model. Unfortunately, all the theories made so far concern the mixed valences and not the Kondo lattice.

(i) Ghatak et al (1976) treat the mixed valences in the alloy analogy (CPA) with the juxtaposition of rare-earth atoms with valences two and three. At low temperatures, the resistivity is found to vary as $N_{\mathrm{d}}^{-2}(\mu, T)$, where $N_{\mathrm{d}}$ is the d electron density of states and $\mu$ the chemical potential. The $\mathrm{f}$ holes are then considered to act like traps on the conducting s electrons as in the semiconductor picture.

(ii) Another approach introduced by Entel et al (1980) is based on the electronphonon interaction related to large changes in the ionic radii when changing valence. Resistivity is found to behave as $[V \mid(E-\mu)]^{2}$, where $V$ is the Anderson s-f hybridisation and $(E-\mu)$ is the distance between the effective position $E$ of the $4 f$ level and the chemical potential, and so decreases with increasing temperature.

(iii) The resistivity of the Anderson lattice has been calculated by Coqblin et al (1980) in the antiferromagnetic case and when the Fermi level lies within the gap created by the antiferromagnetic ordering (semiconductor behaviour) or close to this gap (semi-metal behaviour). By use of the Kubo-Greenwood formula, they found an insulating behaviour for stoichiometric $\mathrm{Tm}_{x} \mathrm{Se}$ and a semi-metallic behaviour off stoichiometry. Difficulties appeared in obtaining a correct Néel temperature and explaining the logarithmic decrease of the resistivity at high temperature.

Therefore, the transport properties in Kondo alloys remain to be found. As observed by Haldane (1977), the way to treat the problem depends on the temperature. At high temperature, interactions between rare-earth atoms become negligible: the system may be treated as a collection of incoherent impurities. At much lower temperatures, coherence develops between virtual bound states and the material behaves as a Kondo lattice.

Section 2 is devoted to a review of the theoretical results obtained for the Kondo lattice. The model used in the following is that introduced by Lacroix and Cyrot (1979): within a functional integration method, they transformed the Kondo interaction into a fictitious $s-f$ hybridisation giving a resonance of width $T_{\mathrm{K}}$ around the impurity level. In a mean field theory, this fictitious hybridisation disappears above a temperature of the order of $T_{K}(5$ or $10 \mathrm{~K})$, the resistivity then being that of free electrons. Experimentally, the resistivity still decreases well above $T_{\mathrm{K}}$ (up to 100 or $200 \mathrm{~K}$ ) and to explain this means going beyond a mean field theory and introducing thermal fluctuations. This is explained at the end of $\$ 2$.

Section 3 deals with the resistivity of the collection of impurities at high temperatures. This calculation is made within the phase shift method. Taking account of thermal fluctuations, the resistivity is found to exhibit a logarithmic decrease.

In $\$ 4$, the resistivity is calculated in the low-temperature limit when the system may be considered as a Kondo lattice. Spatial fluctuations (from site to site) of the $s-f$ hybridisation are considered as being responsible for the electron scattering. The resistivity is found to be either of semiconductor type when the number $n$ of $d$ electrons per impurity is equal to 1 , or of metallic type for $n \neq 1$ : in the latter case, the resistivity starts from zero at $T=0$ (no spatial fluctuation), increases with increasing temperature and passes through a maximum at $T_{\max } \sim(1-n)^{1 / 3} T_{\mathrm{K}}$. 


\section{Kondo lattice}

The Kondo lattice model was first introduced by Doniach (1977) as a generalisation of the Kondo Hamiltonian to the concentrated case. The essential feature lies in the competition between the Kondo effect and magnetic order. In a mean field approximation on a one-dimensional lattice, Doniach (1977) found that the ground state is antiferromagnetic for low $|J| / D$ and is a non-magnetic Kondo singlet for large $|J| / D$. These results were confirmed by Jullien et al (1977) using a renormalisation group treatment.

Lacroix and Cyrot (1979) generalised a method of functional integration to the Kondo lattice first introduced by Yoshimori and Sakurai (1970) for the single impurity case. Using this method, they transformed the Kondo interaction into a fictitious $s-f$ hybridisation. The Hamiltonian obtained is:

$$
\begin{aligned}
H=\sum_{k \sigma}\left(\varepsilon_{k}+\right. & \left.\frac{J}{4}\right) c_{k \sigma}^{\dagger} c_{k \sigma}+\sum_{i \sigma}\left(E_{0}+\frac{J n}{4}\right) d_{i \sigma}^{\dagger} d_{i \sigma}+\frac{J}{2} \sum_{i} x_{i}\left(d_{i \uparrow}^{\dagger} c_{i \uparrow}+c_{i \downarrow}^{\dagger} d_{i \downarrow}\right) \\
& +\frac{J}{2} \sum_{i} y_{i}\left(d_{i \downarrow}^{\dagger} c_{i \downarrow}+c_{i \uparrow}^{\dagger} d_{i \uparrow}\right)
\end{aligned}
$$

where $c_{k}^{\dagger}, d_{i}^{\dagger}$ and $\varepsilon_{k}, E_{0}$ are respectively the creation operators and the energies of an $s$ electron of wavevector $k$ and an f electron on site $i, J$ is the usual Kondo $s-f$ interaction, $n$ is the number of conduction electrons per atom (knowing that there is one impurity per site). $x_{i}$ and $y_{i}$ represent the fictitious $s-f$ hybridisation and are determined self-consistently. They can be considered as an order parameter: their average values are reduced to zero above a temperature $T_{\mathrm{K}}^{*}$. In the following $x_{i}$ will be taken to be equal to $y_{i}$.

With a rectangular conduction band of width $2 D$, they obtained:

$$
\rho_{\mathrm{d}}(\omega)=\frac{1}{8 D} \frac{J^{2} x^{2}}{\left(\omega-E_{0}-\frac{1}{4} J n\right)^{2}} \quad \rho_{\mathrm{c}}(\omega)=\frac{1}{2 D}
$$

for the density of states of the $4 \mathrm{f}$ and conduction electrons where $\omega_{1}<\omega<\omega_{2}$ and $\omega_{3}<\omega<\omega_{4}$ and $\rho_{\mathrm{d}}=\rho_{\mathrm{c}}=0$. Otherwise $\omega_{1}, \omega_{2}, \omega_{3}$ and $\omega_{4}$ are given by:

$$
\begin{aligned}
& \omega_{1}=\frac{1}{2}\left[-D+E_{0}+\frac{1}{4} J(1+n)-\left\{\left[D+E_{0}-\frac{1}{4} J(1-n)\right]^{2}+J^{2} x^{2}\right\}^{1 / 2}\right] \\
& \omega_{2}=\frac{1}{2}\left[+D+E_{0}+\frac{1}{4} J(1+n)-\left\{\left[D-E_{0}+\frac{1}{4} J(1-n)\right]^{2}+J^{2} x^{2}\right\}^{1 / 2}\right] \\
& \omega_{3}=\frac{1}{2}\left[-D+E_{0}+\frac{1}{4} J(1+n)+\left\{\left[D+E_{0}-\frac{1}{4} J(1-n)\right]^{2}+J^{2} x^{2}\right\}^{1 / 2}\right] \\
& \omega_{4}=\frac{1}{2}\left[+D+E_{0}+\frac{1}{4} J(1+n)+\left\{\left[D-E_{0}+\frac{1}{4} J(1-n)\right]^{2}+J^{2} x^{2}\right\}^{1 / 2}\right] .
\end{aligned}
$$

The Kondo temperature $T_{\mathrm{K}} \dagger$ given by the ground state energy has the residual variation $D \exp (1 / \rho J)$, where $\rho$ is the initial density of states at the Fermi level, as for a single impurity. The schematic behaviour of $\rho_{\mathrm{d}}$ and $\rho_{\mathrm{c}}$ is shown in figure 1 . As soon as $x \neq 0$, a gap is opened in the density of states of the order of $T_{\mathrm{K}}$ around the energy of the impurity level: the width of the resonance is of the same order. The phase diagram

+ In this paper we distinguish the temperature $T_{\mathrm{K}}^{*}$ above which the Kondo parameters $x$ and $y$ are equal to zero, and the temperature $T_{K}$ given by the ground state energy. The two temperatures are in fact of the same order. 


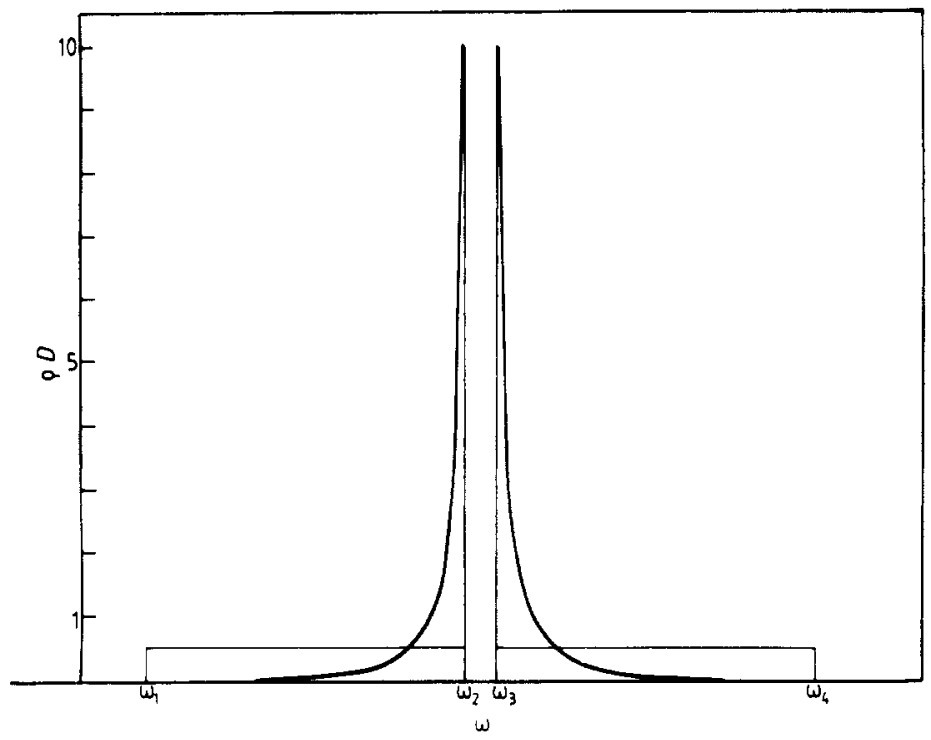

Figure 1. Schematic density of states of conduction and $f$ electrons for the Kondo lattice.

is discussed as a function of $J / D$ and $n$ : the system is magnetic below a critical value of $J / D$; the magnetic order is then ferromagnetic for low values of $n$ and becomes antiferromagnetic when $n$ is close to one. The ground state is found to be insulating when the conduction band is half-filled: $n=1$.

In a mean field theory, as soon as the temperature becomes larger than $T_{\mathrm{K}}^{*}, x$ and $y$ are reduced to zero and the resistivity obtained is that of free electrons. In fact, experiments show that the resistivity exhibits a large decrease from 5 to 100 or $200 \mathrm{~K}$, well above $T_{\mathbf{K}}^{*}(5$ or $10 \mathrm{~K}$ ). To account for this, we must go beyond a mean field theory and introduce thermal fluctuations. The variation of the free energy $F$ as a function of $x$ is given by:

$$
F=-\frac{J x^{2}}{2}+2 \int \omega\left(\rho_{\mathrm{c}}(\omega)+\rho_{\mathrm{d}}(\omega)\right) f(\omega) \mathrm{d} \omega-T S
$$

The results are characteristic of a second-order transition with the presence of a minimum for temperatures lower than $T_{\mathrm{K}}^{*}$. The full curve in figure 2 shows the thermal variation of $x_{\min }$ (with $|J| / D=0.2$ and $n=1$ ). To go beyond a mean field theory one must consider a statistical distribution around the value of the minimum.

In the low-temperature limit, the free energy can be written:

$$
F=-\frac{J x^{2}}{2}\left[1-\frac{J}{2 D}\left(-1+\ln \frac{J^{2} x^{2}}{4 n D^{2}}\right)\right]
$$

exhibiting a minimum for $J^{2} x^{2} / D^{2}=4 n \exp (2 D / J)$ as found by Lacroix and Cyrot (1979).

In the high-temperature limit:

$$
F=\left(J^{2} x^{2} / 4 D\right) \ln \left(T / T_{\mathrm{K}}^{*}\right)-k_{\mathrm{B}} T \ln 2
$$




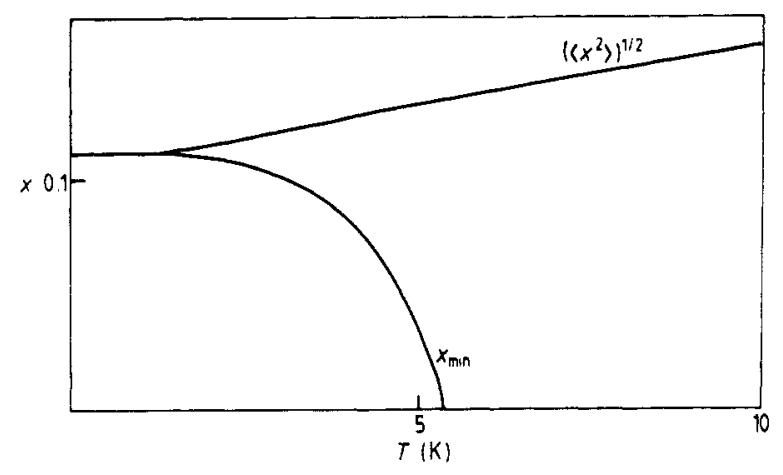

Figure 2. Temperature dependence of $x_{\min }$ and $\left(\left\langle x^{2}\right\rangle\right)^{1 / 2}$.

with

$$
k T_{\mathrm{K}}^{*}=a(n) D \exp (1 / \rho J) \quad a(n)=1.176[n(2-n)]^{1 / 2} \exp [1-(1-n) /(2-n)] .
$$

The variation of the Kondo temperature $T_{\mathbf{K}}^{*}$ with $n$ is shown in figure 3 .

The high-temperature value of $\left\langle x^{2}\right\rangle$ is given by:

$$
\left\langle x^{2}\right\rangle=k T\left[\left(J^{2} / 2 D\right) \ln \left(T / T_{\mathrm{K}}^{*}\right)\right]^{-1} .
$$

Numerator and denominator give opposite contributions with temperature, the former due to the Boltzmann statistics and the latter due to the curvature of the free energy.

In order to appreciate the importance of the thermal fluctuations, we have compared the thermal variation of $x_{\min }$ and $\left(\left\langle x^{2}\right\rangle\right)^{1 / 2}$ in figure 2 . Effectively, the thermal fluctuations are of great importance above $T_{\mathbf{K}}^{*}$ and can considerably modify the resistivity behaviour at high temperatures.

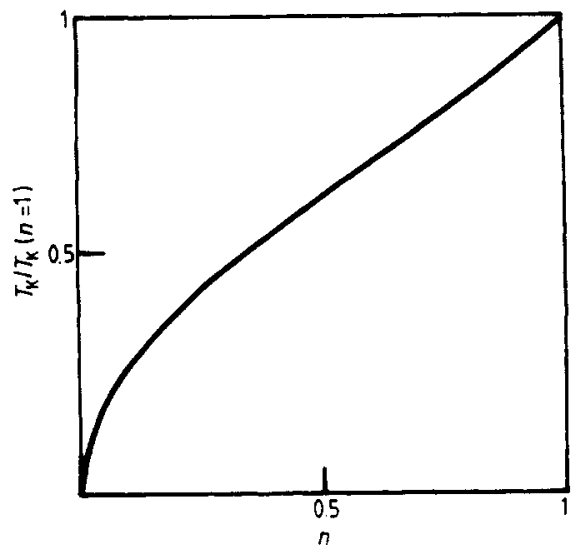

Figure 3. Variation of the Kondo temperature $T_{\mathbf{K}}^{*}$ with $n$.

\section{Resistivity of the collection of impurities at high temperatures}

At high temperatures, all the virtual bound states scatter the conduction electrons in a completely incoherent way. The system can be considered as a collection of incoherent 
impurities. Thermal fluctuations and resistivity are then calculated within the classical phase shift method.

In order to evaluate the thermal fluctuations, we start as before from the expansion of the free energy:

$$
F=-\frac{J x^{2}}{2}+\int_{-D}^{+D} \omega \rho(\omega) f(\omega) \mathrm{d} \omega-T S
$$

The density of states $\rho(\omega)$ of the virtual bound state is directly related to the phase shift $\varphi(\omega)$ introduced by the impurity (Friedel 1954, 1958):

$$
\varphi(\omega)=\tan ^{-1}(\Delta / \omega)
$$

where $\Delta$ is the width of the virtual bound state:

$$
\Delta=\pi\left(\frac{1}{2} J x\right)^{2}(1 / 2 D)
$$

Equation (9) becomes:

$$
F=-\frac{J x^{2}}{2}+\frac{2}{\pi} \int_{-D}^{+D} \omega \frac{\Delta}{\omega^{2}+\Delta^{2}} f(\omega)-T S
$$

At high temperatures, the result is similar to the high-temperature limit of the Kondo lattice with the same comments on the thermal variation of $\left\langle x^{2}\right\rangle$ :

$$
F=\left(J^{2} x^{2} / 4 D\right) \ln \left(T / T_{\mathrm{K}}^{*}\right)-k_{\mathrm{B}} T \ln 2
$$

with $k T_{\mathrm{K}}^{*}=1.14 D \exp (2 D / J)$.

Considering that the potential responsible for the scattering of the conduction electrons is the fictitious $\mathrm{s}-\mathrm{f}$ hybridisation $\frac{1}{2} J x$, the resistivity is then calculated within the phase shift method (Friedel 1954, 1958):

$$
\rho(\omega)=\left(4 \pi / n e^{2} k_{\mathrm{F}}\right) \sin ^{2} \varphi(\omega)
$$

Then, writing $\sigma=\int(-\mathrm{d} f / \mathrm{d} \omega) \sigma(\omega) \mathrm{d} \omega$, we find:

$$
\rho=\left(4 \pi / n e^{2} k_{\mathrm{F}}\right)\left[3 J^{4} x^{4} / 16 D^{2}(k T)^{2}\right] .
$$

Taking account of thermal fluctuations to evaluate $x^{4}$, the resistivity behaves at high temperatures as:

$$
\rho=\left(4 \pi / n e^{2} k_{\mathrm{F}}\right) 9\left[4 \ln ^{2}\left(T / T_{\mathrm{K}}\right)\right]^{-1} .
$$

Writing $\ln \left(T / T_{\mathrm{K}}\right)=\left[\ln (T / D)-\ln \left(T_{\mathrm{K}} / D\right)\right]$, we find for $T \gg T_{\mathrm{K}}$ the classical Kondo expression:

$$
\rho=\left(4 \pi / n e^{2} k_{\mathrm{F}}\right) \frac{9}{4}(J / 2 D)^{2}[1+(J / D) \ln (k T / D)] .
$$

The resistivity exhibits Kondo behaviour at high temperatures within a logarithmic decrease in $J \ln (k T / D)$. This permits the high-temperature behaviour of many concentrated compounds of anomalous rare earths to be explained. In fact, the resistivity does not reach the zero value at high temperature due to the effects of phonons and impurities. 


\section{Resistivity of the Kondo lattice at low temperatures}

At low temperatures, coherence develops between the impurities, and the system may be described as a Kondo lattice. If the Kondo parameter $x$ is uniform on all the sites, the Hamiltonian (1) can easily be diagonalised and nothing else is able to scatter electrons. On the other hand, if we consider the spatial fluctuations (from site to site) of the $s-f$ hybridisation, the following potential remains:

$$
V=\sum_{i} \frac{J \delta_{i}}{2}\left(d_{i \uparrow}^{\dagger} c_{i \uparrow}+c_{i \downarrow}^{\dagger} d_{i \downarrow}+d_{i \downarrow}^{\dagger} c_{i \downarrow}+c_{i \uparrow}^{\dagger} d_{i \uparrow}\right)
$$

with

$$
\delta_{i}=x_{i}-\left\langle x_{i}\right\rangle .
$$

Among all the possible mechanisms (phonons,...), spatial fluctuations are retained here to explain the electron scattering. Let us remark that $\left\langle x_{i}\right\rangle=0$ at high temperature and the potential $V$ is the same as that considered in the previous section.

The relaxation time is calculated by means of the $T$ matrix related to $V$ :

$$
\tau_{i}(\omega)^{-1}=-\operatorname{Im} T_{\mathrm{ss}}
$$

(sd and dd conduction electrons are negligible).

If we express $T_{\mathrm{ss}}$ as a function of the Green function, we find

$$
\tau_{i}(\omega)^{-1}=\left(\frac{1}{2} J \delta_{i}\right)^{2} \rho_{\mathrm{d}}(\omega)
$$

While all the calculations, up to now, have been carried out successfully with a rectangular conduction band, it appears that, for transport properties, such a band does not give a continuous passage from metal to insulator when $n$ tends to one, due to a discontinuous variation of $\rho\left(E_{\mathrm{F}}\right)$, when $E_{\mathrm{F}} \rightarrow \omega_{2}$ (figure 1). To overcome this difficulty, an elliptic band is taken in the following discussion. In the appendix we give the main results for the elliptic band: position of the Fermi level, determination of $T_{\mathrm{K}}$, evaluation of the spatial fluctuations $\left\langle\delta_{i}^{2}\right\rangle \ldots$. There is no qualitative difference from the rectangular band, only some different numerical factors. The most significant change is the behaviour of the density of states at the Fermi level: $\rho_{\mathrm{c}}\left(E_{\mathrm{F}}\right) \sim(1-n)^{2 / 3}$ exhibiting a continuous passage when $n \rightarrow 1$.

In the following resistivity calculation, we distinguish the metallic case (with $n$ close to one) from the insulating case (with $n=1$ ).

\subsection{Resistivity in the metallic case $(n \leqslant 1)$}

As pointed out by Wilson (1953), the expression for the conductivity is:

$$
\sigma=\frac{2}{3} e^{2} \int_{\omega_{1}, \omega_{3}}^{\omega_{2}, \omega_{4}}\left(-\frac{\mathrm{d} f}{\mathrm{~d} \omega}\right) \rho_{\mathrm{c}}(\omega) v^{2} \tau \mathrm{d} \omega
$$

For $n$ close to one, only the states in the $\omega_{2}$ neighbourhood are important in considering the resistivity as $E_{\mathrm{F}}$ is close to $\omega_{2}$. For these states, the band is quasi-parabolic and we can write, for $\omega \sim \omega_{2}, \omega_{2}-\omega=\frac{1}{2} m^{*} v^{2}$. The expression for the effective mass $m^{*}$ 
is given in the appendix. Thus

$$
\begin{gathered}
\sigma=\frac{4 e^{2}}{3 m^{*}} \frac{1}{\left(\frac{1}{2} J \delta\right)^{2}\left(\frac{1}{2} J x\right)^{2}} \int_{\omega_{1}, \omega_{3}}^{\omega_{2,}, \omega_{4}}\left(-\frac{\mathrm{d} f}{\mathrm{~d} \omega}\right)\left(\omega_{2}-\omega\right)\left(\omega-E_{0}-\frac{1}{4} J n\right)^{2} \mathrm{~d} \omega \\
=\sigma(0)\left[1+\left(T / T_{1}\right)^{2}\right] .
\end{gathered}
$$

At very low temperature $\left(T<T_{\mathrm{K}} / 3\right)$, figure 2 shows that $\left\langle x^{2}\right\rangle$ is not very different from $x_{\min }(T=0)$ and we can replace $x$ in equation (21) by its minimum value at $T=0 \mathrm{~K}$. In this way, we find:

$$
\sigma(0)=\frac{2 e^{2}}{3 m^{*} D} \frac{4}{J^{2} \delta^{2}}\left(\frac{3 \pi}{4}(1-n)\right)^{2 / 3}\left(\frac{J^{2} x^{2}}{4 D}\right)^{2} .
$$

In this expression we recognise the classical formula $N_{h} e^{2} \tau / m^{*}$ obtained every time the electrons behave as though they are completely free. Knowing that $\left\langle\delta^{2}\right\rangle$ is in $k T / 2|J|$ (see the appendix), this term gives a linear contribution to the resistivity:

$$
\rho(0)=\left(\frac{3 D}{2(1-n)^{2} T_{\mathrm{K}}^{4}}\right)^{1 / 3} \frac{1}{2 e^{2}}|J| \frac{T}{T_{\mathrm{K}}} .
$$

The expression for $T_{1}$ is:

$$
T_{1}=0.367 T_{\mathrm{K}}(1-n)^{1 / 3} .
$$

The resistivity exhibits a maximum at the same temperature $T_{1}$. The thermal variation of the resistivity at low temperature and for $n \neq 1$ is represented in figure 4 . It is of metallic type with increasing resistivity as the temperature increases. The temperature at which the resistivity passes through a maximum is of the order of $(1-n)^{1 / 3} T_{\mathrm{K}}$. The value of the maximum varies then as $1 /(1-n)^{1 / 3}$, becoming infinite for $n=1$.

\subsection{Resistivity in the semiconductor case $(n=1)$}

$E_{0}$ and $E_{\mathrm{F}}$ take the values 0 and $J / 4$ respectively ( $E_{\mathrm{F}}$ being at the middle of the gap). The semiconductor case is treated for low enough temperatures by writing the Fermi function as

$$
\begin{array}{ll}
1-\exp \left(\beta \omega^{\prime}\right) & \text { for } \beta \omega^{\prime}<0 \\
\exp \left(-\beta \omega^{\prime}\right) & \text { for } \beta \omega^{\prime}>0
\end{array}
$$

Using this in the expression for the conductivity, we find:

$$
\sigma=\frac{16 e^{2}}{3 m^{*}} \frac{k T}{D} \frac{J^{2} x^{2}}{4 D} \frac{\exp \left[-\beta\left(J^{2} x^{2}\right) / 4 D\right]}{\left(\frac{1}{2} J \delta\right)^{2}} .
$$

The thermal variation of the resistivity is of semiconductor type (infinite value at $T=0$ ) with an exponential decrease in $\exp T_{\mathrm{K}} / T$. Figure 4 shows the resistivity behaviour at low temperature in the semiconductor case. 


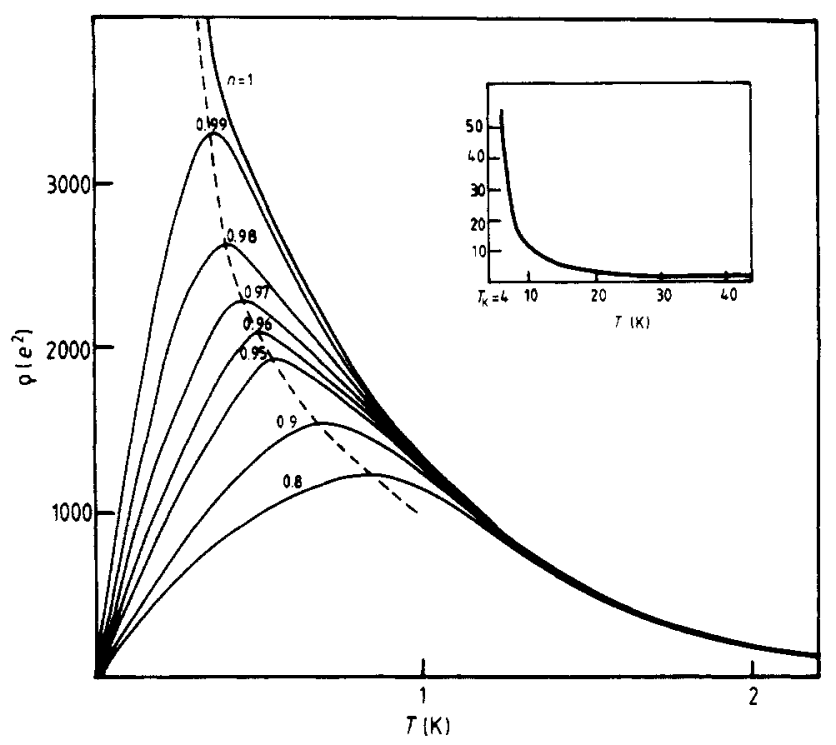

Figure 4. Temperature dependence of resistivity for various values of $n$.

\section{Conclusion}

In conclusion, the treatment of the system as an impurity collection at high temperatures leads to a logarithmic behaviour of the resistivity typical of a Kondo system. At low temperatures, we are dealing with a Kondo lattice and the resistivity is either of metallic type for $n \neq 1\left(\mathrm{CeAl}_{2}, \ldots\right)$ or of semiconductor type (TmSe stoichiometric, ...). For the first case, the resistivity theoretically tends to zero for $T=0$; in fact, this result is unrealistic and a residual resistivity always remains due to some impurities. The thermal variation of the resistivity is found to be linear, but the fact that a self-consistent calculation of the fluctuations would give a $T^{2}$ variation is not excluded. No discontinuity at all is observed at $T_{\mathrm{K}}$. Contrary to Coqblin et al (1980), the resistivity passes through a maximum at a temperature of the order of $T_{K}$ and exhibits logarithmic behaviour at high temperature. Finally, as the conductivity $\sigma(\omega)$ varies rapidly with $\omega\left(\sigma(\omega) \sim\left(\omega_{2}-\omega\right)\left(\omega-E_{0}-\frac{1}{4} J n\right)^{2}\right)$, we expect strong values of the thermopower in agreement with the experimental results (Jaccard et al 1979).

\section{Appendix. Determination of the parameters for an elliptic band}

In this appendix, we calculate the parameters for an initial elliptic band, following the same method as Lacroix and Cyrot (1979). We start with the following density of states for the conduction band:

$$
\rho_{0}(\omega)=\left(2 / \pi D^{2}\right)\left(D^{2}-\omega^{2}\right)^{1 / 2} .
$$


Then the Green functions of the Kondo lattice are:

$$
\begin{aligned}
& G_{\mathrm{ss}}(\omega)=\frac{2}{D^{2}}\left\{\omega-\frac{J}{4}-\frac{J^{2} x^{2}}{4\left(\omega-E_{0}-\frac{1}{4} J n\right)}-\left[\left(\omega-\frac{J}{4}-\frac{J^{2} x^{2}}{4\left(\omega-E_{0}-\frac{1}{4} J n\right)}\right)^{2}-D^{2}\right]^{1 / 2}\right\} \\
& G_{\mathrm{dd}}(\omega)=\frac{1}{\omega-E_{0}-\frac{1}{4} J n}+\frac{J^{2} x^{2}}{4\left(\omega-E_{0}-\frac{1}{4} J n\right)^{2}} G_{\mathrm{ss}}(\omega) \\
& G_{\mathrm{sd}}(\omega)=\frac{J x}{2\left(\omega-E_{0}-\frac{1}{4} J n\right)} G_{\mathrm{ss}}(\omega) .
\end{aligned}
$$

From these expressions, we can deduce the density of states for the conduction and f electrons:

$$
\begin{aligned}
& \rho_{\mathrm{c}}(\omega)=\frac{2}{\pi D^{2}} \frac{\left[\left(\omega-\omega_{1}\right)\left(\omega_{2}-\omega\right)\left(\omega_{3}-\omega\right)\left(\omega_{4}-\omega\right)\right]^{1 / 2}}{\left(\omega-E_{0}-\frac{1}{4} J n\right)} \\
& \rho_{\mathrm{d}}(\omega)=\frac{J^{2} x^{2}}{4\left(\omega-E_{0}-\frac{1}{4} J n\right)^{2}} \rho_{\mathrm{c}}(\omega)
\end{aligned}
$$

when $\omega_{1}<\omega<\omega_{2}$ and $\omega_{3}<\omega<\omega_{4} .\left(\omega_{1}, \omega_{2}, \omega_{3}\right.$ and $\omega_{4}$ are the same as in the case of a rectangular density of states (see equation (3)).)

In the following, we restrict ourselves to values of $n$ of the order of one $(n \leqslant 1)$; thus the Fermi level will be in the lower sub-band very near $\omega_{2}$.

\section{A.l. Position of the Fermi level}

The total number of electrons is equal to $1+n$. Thus we can write:

$$
1+n=2 \int_{\omega_{1}}^{E_{\mathrm{F}}}\left(\rho_{\mathrm{c}}(\omega)+\rho_{\mathrm{d}}(\omega)\right) \mathrm{d} \omega
$$

If we put $u=\omega-\frac{1}{4} J-\left\{J^{2} x^{2}\left[4\left(\omega-E_{0}-\frac{1}{4} J n\right)\right]^{-1}\right\}$ this integral becomes:

$$
1+n=\frac{4}{\pi D^{2}} \int_{-D}^{D-\varepsilon}\left(D^{2}-u^{2}\right)^{1 / 2} \mathrm{~d} u
$$

with

$$
D-\varepsilon=E_{\mathrm{F}}-\frac{1}{4} J-\left\{J^{2} x^{2}\left[4\left(E_{\mathrm{F}}-E_{0}-\frac{1}{4} J n\right)\right]^{-1}\right\} .
$$

With the condition in equation (A.5), $\varepsilon \rightarrow 0$ when $n \rightarrow 1$. We can develop equation (A.4) with respect to $\varepsilon$ and we obtain:

$$
1+n=2+(4 / 3 \pi)(2 \varepsilon / D)^{3 / 2}
$$

Using equation (A.5) and the fact that $\omega_{2}-E_{0}-\frac{1}{4} J n \sim\left(J^{2} x^{2} / 4 D\right)$ for $n \simeq 1$, we 
finally obtain:

$$
\omega_{2}-E_{\mathrm{F}}=(3 \pi / 4)^{2 / 3}(1-n)^{2 / 3}\left(J^{2} x^{2} / 8 D\right) .
$$

The density of states at the Fermi level is given by:

$$
\rho_{\mathrm{c}}\left(E_{\mathrm{F}}\right)=(2 / \pi D)(3 \pi / 4)^{1 / 3}(1-n)^{1 / 3} .
$$

$\rho_{\mathrm{c}}\left(E_{\mathrm{F}}\right)$ and $\rho_{\mathrm{d}}\left(E_{\mathrm{F}}\right)$ go continuously to zero when $n \rightarrow 1$.

\section{A.2. Number of holes in the conduction band}

As this number plays an important role in the conductivity, we give the expression for it here:

$$
N_{\mathrm{h}}=2 \int_{E_{\mathrm{F}}}^{\omega_{2}} \rho_{\mathrm{c}}(\omega) \mathrm{d} \omega
$$

Near $\omega_{2}$, the expression for $\rho_{\mathrm{c}}$ (equation (A.2)) becomes:

$$
\rho_{\mathrm{c}}(\omega)=(2 / \pi D)\left(2 D / J^{2} x^{2}\right)^{1 / 2}\left(\omega_{2}-\omega\right)^{1 / 2} .
$$

Substituting equation (A.9) into equation (A.8) we obtain

$$
N_{\mathrm{h}}=(1-n)\left(J^{2} x^{2} / 4 D^{2}\right) \text {. }
$$

From the above expression for $\rho_{c}(\omega)$, we can deduce the value of the effective mass for the holes of the conduction band, which is involved in the resistivity (equation (23)): if the effective mass of the carriers is $m^{*}$, the density of states is:

$$
\rho_{\mathrm{c}}(\omega)=\left(1 / 2 \pi^{2}\right)\left(2 m^{*}\right)^{3 / 2}\left(\omega_{2}-\omega\right)^{1 / 2}
$$

Comparing this with equation (A.9), we obtain:

$$
m^{*}=\frac{1}{2}\left(2 D / J^{2} x^{2}\right)^{1 / 3}(8 \pi / D)^{2 / 3} .
$$

\section{A.3. Kondo temperature for $n=1$}

As the Kondo temperature has no singularity for $n \rightarrow 1$, we can use the value of $T_{\mathrm{K}}$ for $n=1$ in all the expressions. For $n=1, E_{0}=0$ and $E_{\mathrm{F}}=J / 4$.

The free energy at $T=0 \mathrm{~K}$ is given by:

$$
F=-\frac{J x^{2}}{2}+2 \int_{\omega_{1}, \omega_{3}}^{\omega_{2}, \omega_{4}} \omega\left(\rho_{\mathrm{c}}(\omega)+\rho_{\mathrm{d}}(\omega)\right) \mathrm{d} \omega
$$

As $\omega_{1}=-\omega_{4}$ and $\omega_{2}=-\omega_{3}$, equation (A.12) can be reduced to elliptic integrals:

$$
F=-\frac{J x^{2}}{2}-\frac{4}{\pi D^{2}} \int_{\omega_{3}}^{\omega_{4}}\left[\left(\omega_{4}^{2}-\omega^{2}\right)\left(\omega^{2}-\omega_{3}^{2}\right)\right]^{1 / 2}\left(1+\frac{J^{2} x^{2}}{4 \omega^{2}}\right) \mathrm{d} \omega
$$


The parameter of these elliptic integrals is:

$$
q=\left(\omega_{2}^{2}-\omega_{3}^{2}\right)^{1 / 2} / \omega_{4} \simeq 1-\frac{1}{2}\left(\omega_{3} / \omega_{4}\right)^{2} .
$$

Using the formula given by Gradshteyn and Ryshik (1965) we obtain

$$
F=-\frac{1}{2} J x^{2}-\left(4 / \pi D^{2}\right)\left[\frac{1}{3} \omega_{4}^{3} E(q)-\left(\frac{2}{3} \omega_{4} \omega_{3}^{2}-\frac{1}{4} \omega_{4} J^{2} x^{2}\right) K(q)\right]
$$

where $K(q)$ and $E(q)$ are the elliptic integrals of the first and second kind respectively.

For $q$ close to $1, E(q)$ and $K(q)$ take the following forms:

$$
\begin{aligned}
& E(q)=1+\frac{1}{2}\left(1-q^{2}\right) \ln 4\left(1-q^{2}\right)^{-1 / 2} \\
& K(q)=\ln 4\left(1-q^{2}\right)^{-1 / 2} .
\end{aligned}
$$

Equation (A.13) becomes:

$$
\Delta F=-\frac{1}{2} J x^{2}+\left(J^{2} x^{2} / \pi D\right) \ln \left(J^{2} x^{2} / 16 D^{2}\right)
$$

Minimising this expression, we obtain:

$$
J^{2} x^{2} / 16 D^{2}=\exp (\pi D / 2 J)
$$

Thus, the Kondo temperature, which is proportional to $J^{2} x^{2} / D$, varies as $\exp (1 / \rho J)$, where $\rho$ is the density of states at the Fermi level for the initial conduction band $(\rho=2 / \pi D)$.

\section{A.4. Calculation of $\left\langle\delta^{2}\right\rangle$ for $n=1$}

The free energy of the Kondo lattice can be written in the most general way (Lacroix and Cyrot 1979):

$$
F=-\frac{J}{2} \sum_{i} x_{i}^{2}-\frac{1}{\beta} \operatorname{Tr} \ln \left(1-V G^{0}\right)
$$

where:

$$
\begin{aligned}
x_{i} & =x+\delta_{i} \quad\left(\left\langle\delta_{i}\right\rangle=0\right) \\
V & =\tilde{V}+\sum v_{i} \\
\tilde{V} & =\frac{J x}{2} \sum_{i \sigma}\left(c_{i \sigma}^{\dagger} d_{i \sigma}+\mathrm{CC}\right) \\
v_{i} & =\frac{J \delta_{i}}{2} \sum_{i \sigma}\left(c_{i \sigma}^{+} d_{i \sigma}+\mathrm{CC}\right) .
\end{aligned}
$$

For small values of $\delta_{i}$, equation (A.17) becomes:

$$
F \simeq F_{0}(x)-\frac{J}{2} \sum_{i} \delta_{i}^{2}+\frac{1}{2 \beta} \sum_{i} \operatorname{Tr}\left(v_{i} G\right)^{2}
$$


where $G$ is the Green function of the Kondo lattice (equation (A.1)). At $T=0 \mathrm{~K}$, equation (A.18) becomes:

$$
\frac{1}{2 \beta} \sum_{i} \operatorname{Tr}\left(v_{i} G\right)^{2}=\frac{J^{2}}{2 \pi} \sum_{i} \delta_{i}^{2} \int_{\omega_{1}}^{\omega_{2}} \operatorname{Im}\left(G_{\mathrm{sd}}^{2}(\omega)+G_{\mathrm{ss}}(\omega) G_{\mathrm{dd}}(\omega)\right) \mathrm{d} \omega .
$$

Here again we have to calculate elliptic integrals.

Using the formulae of Gradshteyn and Ryshik (1965) we obtain the variation of the free energy per atom due to the fluctuations of the parameter $x_{i}$ :

$$
\Delta F=-\frac{1}{2} J \delta^{2}-\left(J^{2} \delta^{2} / \pi D\right) \ln \left(J^{2} x^{2} / 16 D^{2}\right) .
$$

Thus, using equation (A.16), at $T=0 \mathrm{~K}$, we have $\Delta F=-J \delta^{2}$. The thermal mean value $\left\langle\delta^{2}\right\rangle$ can be calculated using:

$$
\left\langle\delta^{2}\right\rangle=\int \delta^{2} \exp (-\beta \Delta F) \mathrm{d} \delta\left(\int \exp (-\beta \Delta F) \mathrm{d} \delta\right)^{-1}
$$

At low temperatures we find:

$$
\left\langle\delta^{2}\right\rangle=k T / 2|J|
$$

At high temperatures, this method is not valid as equation (A.20) is the free energy at $T=0 \mathrm{~K}$. Above the Kondo temperature $\left\langle\delta^{2}\right\rangle=\left\langle x^{2}\right\rangle$ and the thermal fluctuations of $x^{2}$ have been calculated in $\$ 3$ for high temperatures.

\section{References}

Coqblin B, Bhattacharjee A K and Jullien R 1980 J. Magn. Magn. Mater. 15-18 995

Doniach S 1977 Physica B 91231

Entel P, Mühlschlegel B and Ono Y 1980 Z. Phys. B 38227

Friedel J 1954 Adv. Phys. 3446

-1958 Nuovo Cimento Suppl. 7287

Ghatak S K, Avignon M and Bennemann K H 1976 J. Phys. F: Met. Phys. 61441

Gradshteyn I S and Ryshik I M 1965 Tables of Integrals, Series and Products (New York: Academic)

Haldane F D M 1977 Valence Instabilities and Narrow Band Phenomena ed R D Parks (New York: Plenum) p 191

Jaccard D. Sierro J and Bucher E 1979 Solid State Commun. 31713

Jullien R, Fields J N and Doniach S 1977 Phys. Ret. B 164889

Lacroix C and Cyrot M 1979 Phys. Rev. B 201969

Lawrence J M, Riseborough P S and Parks R D 1981 Rep. Prog. Phys. 4430

Wilson A H 1953 The Theory of Metals (London: Cambridge University Press)

Yoshimori A and Sakurai A 1970 Prog. Theor. Phys. Suppl. 46162 